\title{
Modification of resonance Raman scattering in very intense laser fields
}

\author{
Claude Cohen-Tannoudji and Serge Reynaud \\ Laboratoire de Spectroscopie Hertzienne, Ecole Normale Supérieure et Collège de France, \\ 24 rue Lhomond, 75231 Paris Cedex 05, France
}

Received 22 June 1976, in final form 22 September 1976

\begin{abstract}
The general method developed in a previous paper is applied to the problem of resonance Raman scattering in very intense laser fields. At very low intensities the spectrum consists of three monochromatic lines (Rayleigh, Raman Stokes and anti-Stokes) which split into a triplet and two doublets when the laser light, tuned on one of the two transitions connecting the upper state to the two lower sublevels, has a sufficiently high intensity to saturate this transition but not the second one. The Raman and Rayleigh lines are completely mixed when the intensity becomes so high that both transitions are saturated. The corresponding evolution of the spectrum is determined. The widths and intensities of the various components are calculated in the limit of very high intensity. Optical-pumping effects leading to asymmetries in the fluorescence spectrum are taken into account. It is shown that they are responsible for a decrease of the total scattered light at very high intensities and for an accumulation of atoms in a well defined coherent superposition of the two lower sublevels. The absorption rate for photons from a weak probe tunable laser beam is also evaluated.
\end{abstract}

\section{Introduction}

The purpose of this paper is to calculate the modifications to Raman scattering at very high intensities.

We consider the simplest possible case of an atom having two sublevels $g$ and $g^{\prime}$ in the ground state separated by a splitting $s$ and a single excited level $e$ (see figure 1) $\dagger$. As in resonance fluorescence experiments (Schuda et al 1974, Walther 1975, Wu et al 1975) a beam of such atoms is supposed to be irradiated at right angles by a single-mode laser of frequency $\omega_{\mathrm{L}}$ near the frequency $\omega_{0}$ of the $e \rightarrow g$ atomic transition and the spectral distribution of the fluorescence light is observed in the third perpendicular direction, so that the Doppler effect is avoided. The polarization of the laser light is such that both transitions $e \rightarrow g$ and $e \rightarrow g^{\prime}$ are excited. The problem is to study the modification of the fluorescence and absorption spectra at very high intensities of the incident light.

$\uparrow$ Such a situation occurs, for example, for a $J_{g}=\frac{1}{2} \rightarrow J_{e}=\frac{1}{2}$ transition, the splitting between the two sublevels $|g\rangle=\left|+\frac{1}{2}\right\rangle$ and $\left|g^{\prime}\right\rangle=\left|-\frac{1}{2}\right\rangle$ of the ground state being produced by a static magnetic field. The Larmor frequency in the upper state (electronic Landé $g$ factor) is supposed to be very large compared to the Larmor frequency in the ground state (no electronic paramagnetism, nuclear $g$ factor) so that by adjusting the laser frequency near the frequency $\omega_{0}$ of the transition $e \rightarrow g$, one can forget the other excited sublevel $e^{\prime}$ which is too far away. 


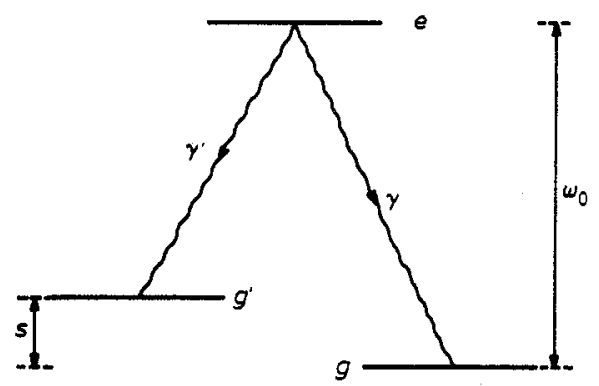

Figure 1. Energy-level diagram of the three-level atom. $s$ is the splitting of the lower state, $\omega_{0}$ the frequency of the $e \rightarrow g$ transition, $\gamma$ and $\gamma^{\prime}$ the radiative transition probabilities for the two transitions $e \rightarrow g, e \rightarrow g^{\prime}$.

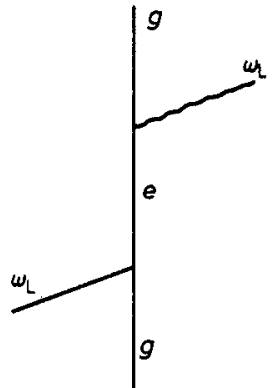

(a)

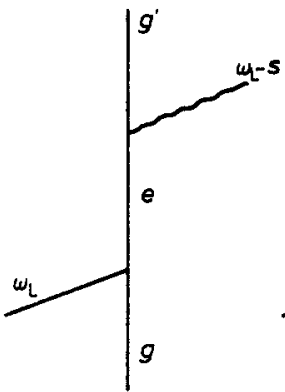

(b)

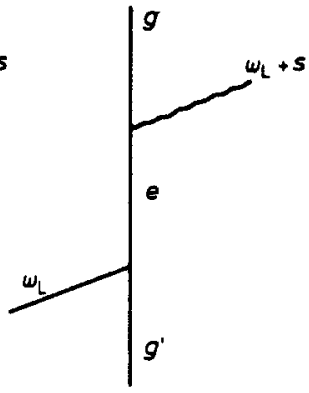

(c)

Figure 2. Lowest order Feynman diagrams describing (a) Rayleigh, (b) Raman Stokes' and (c) Raman anti-Stokes' scatterings.

Let us first recall the well known lowest order perturbative results associated with the three Feynman diagrams of figure 2. Diagram 2(a) describes elastic Rayleigh scattering, the frequency of the fluorescence photons being equal to $\omega_{\mathrm{L}}$, while diagrams $2(b)$ and $2(c)$ describe inelastic Raman Stokes' $\left(\omega=\omega_{\mathrm{L}}-s\right)$ and Raman antiStokes' $\left(\omega=\omega_{\mathrm{L}}+s\right)$ scatterings, respectively. These predictions concerning the fluorescence spectrum remain valid as long as

$$
\omega_{1} \ll \Gamma \text { or } \delta
$$

where $\omega_{1}$ is the Rabi nutation frequency characterizing the coupling of the laser with the $e \rightarrow g$ transition, $\Gamma$ the natural width of $e$ and

$$
\delta=\omega_{0}-\omega_{\mathrm{L}}
$$

the detuning between the laser and atomic frequencies.

When the laser intensity becomes sufficiently large so that

$$
\Gamma, \delta \ll \omega_{1} \ll s
$$

the previous results are modified in a way which can be easily understood from the characteristics of the dynamical Stark effect on a two-level atom. The condition $\omega_{1} \gg \Gamma, \delta$ requires a treatment to all orders of the coupling of the laser with the saturated $e \rightarrow g$ transition. However, as $s$ remains large compared to $\omega_{1}$, the nonresonant coupling with the $e \rightarrow g^{\prime}$ transition has a very weak effect and may be ignored (or treated to the lowest order for the anti-Stokes' line). This explains why 
the Rayleigh line becomes a triplet centred on $\omega_{\mathrm{L}}$ with a splitting determined by $\omega_{1}$ while the Raman lines become two doublets around $\omega_{\mathrm{L}}-s$ and $\omega_{\mathrm{L}}+s$ with the same splitting. The doublet structure of the Raman Stokes' line may be interpreted as a manifestation of the Autler-Townes splitting on the $e \rightarrow g^{\prime}$ transition (Autler and Townes 1955, Mollow 1972, Baklanov 1973).

When the light intensity becomes so large that

$$
\omega_{1} \gg s, \delta, \Gamma
$$

both transitions are saturated and the clear separation between Rayleigh-type and Raman-type processes is no longer possible.

In this paper we try to give a quantitative description of the fluorescence and absorption spectra in the two regimes corresponding to conditions (1.3) and (1.4) and to discuss the evolution of such spectra when the light intensity increases gradually from low values (condition (1.1)) to very high values (condition (1.4)).

We will closely follow the theoretical method presented in Cohen-Tannoudji and Reynaud (1977, to be referred to as I). The general idea is to consider the total system atom + laser mode as a closed system (dressed atom); resonance fluorescence then appears as spontaneous emission from such a system. The method of $I$ allows a quantitative calculation of the widths and intensities of the various components of the fluorescence and absorption spectra. In resonant Raman scattering, opticalpumping effects are important since they can cause an accumulation of atoms in one of the two sublevels of the ground state (or in a coherent superposition of them). It follows that some populations are very small in the steady-state regime and that the contribution of the transient regime may be important. The method of I which allows a clear separation between steady-state and transient regime contributions is very convenient for studying these effects which are at the origin of important asymmetries in the fluorescence spectrum.

The polarization of the fluorescence photons can also be determined; this allows us to understand the variation of the fluorescence spectrum with the detection polarization.

\section{Notation}

The two transitions $e \rightarrow g, e \rightarrow g^{\prime}$ (see figure 1) correspond to two orthogonal polarizations $\hat{\boldsymbol{\epsilon}}, \hat{\boldsymbol{\epsilon}}^{\prime}$. The matrix elements of the atomic dipole operator $D$ are

$$
\begin{aligned}
& \langle g|\boldsymbol{D}| e\rangle=d \hat{\boldsymbol{\epsilon}} \\
& \left\langle g^{\prime}|\boldsymbol{D}| e\right\rangle=d^{\prime} \hat{\boldsymbol{\epsilon}}^{\prime} .
\end{aligned}
$$

The spontaneous transition rates $e \rightarrow g$ and $e \rightarrow g^{\prime}$ are therefore given by $\dagger$

$$
\gamma=|d|^{2} \quad \gamma^{\prime}=\left|d^{\prime}\right|^{2}
$$

The polarization $\hat{\epsilon}_{\mathrm{L}}$ of the laser field is generally a linear superposition of $\hat{\boldsymbol{\epsilon}}, \hat{\boldsymbol{\epsilon}}^{\prime}$ (and $\hat{\boldsymbol{\epsilon}}^{\prime \prime}$ which forms an orthonormal basis with $\hat{\boldsymbol{\epsilon}}$ and $\left.\hat{\boldsymbol{\epsilon}}^{\prime}\right)_{+}^{+}$

$$
\hat{\boldsymbol{\epsilon}}_{\mathrm{L}}=\lambda \hat{\boldsymbol{\epsilon}}+\mu \hat{\boldsymbol{\epsilon}}^{\prime}+\nu \hat{\boldsymbol{\epsilon}}^{\prime \prime}
$$

$\dagger$ Various factors appearing in the definition of $\gamma, \gamma^{\prime}(2 \pi$, density of the final states, ...) are reincluded in the definition of $D$ in order to get simple expressions for $\gamma, \gamma^{\prime}$.

$\ddagger$ The polarization $\hat{\boldsymbol{\epsilon}}^{\prime \prime}$ appears in other atomic transitions connecting $g$ or $g^{\prime}$ to other excited sublevels $e^{\prime}$ far from $e$. It plays no role in our problem except that $\left.\left|\lambda_{\mid}^{2}+\right| \mu\right|^{2}=1-|v|^{2}$ can vary with $\hat{\boldsymbol{\epsilon}}_{\mathrm{L}}$. 
The frequencies $\omega_{1}$ and $\omega_{1}^{\prime}$ which characterize the coupling with the laser on the transitions $e \rightarrow g, e \rightarrow g^{\prime}$ are proportional to $\lambda d$ and $\mu d^{\prime}$, respectively. It is always possible to choose the relative phases of $g$ and $g^{\prime}$ with respect to $e$ so that $\omega_{1}$ and $\omega_{1}^{\prime}$ are real and positive. The coupling Hamiltonian $V$ between the atom and the laser mode can be written (in the rotating-wave approximation)

$$
\begin{aligned}
V=\frac{\omega_{1}}{2} \sum_{n}(|e, n\rangle\langle g, n+1|+| g, n+1\rangle\langle e, n|) \\
+\frac{\omega_{1}^{\prime}}{2} \sum_{n}\left(|e, n\rangle\left\langle g^{\prime}, n+1|+| g^{\prime}, n+1\right\rangle\langle e, n|\right) .
\end{aligned}
$$

$V$ describes transitions from $g$ or $g^{\prime}$ to $e$ (or vice versa), the number $n$ of photons in the laser mode decreasing (or increasing) by one unit. Strictly speaking, $\omega_{1}$ and $\omega_{1}^{\prime}$ are proportional to $/ n$, but this variation can be ignored, as a consequence of the quasi-classical character of laser mode states (see I, $\$ \$ 2$ and 4.1). If $\hat{\boldsymbol{\epsilon}}_{\mathrm{L}}$ is orthogonal to $\hat{\boldsymbol{\epsilon}}\left(\hat{\boldsymbol{\epsilon}}^{\prime}\right), \omega_{1}\left(\omega_{1}^{\prime}\right)$ vanishes

$$
\begin{aligned}
& \hat{\boldsymbol{\epsilon}} \cdot \hat{\boldsymbol{\epsilon}}_{\mathrm{L}}=0 \Rightarrow \omega_{1}=0 \\
& \hat{\boldsymbol{\epsilon}}^{\prime} \cdot \hat{\boldsymbol{\epsilon}}_{\mathrm{L}}=0 \Rightarrow \omega_{1}^{\prime}=0 .
\end{aligned}
$$

One can detect the fluorescence light emitted with the detection polarization $\hat{\boldsymbol{\epsilon}}_{\mathrm{D}}$, or without a polarization analyser.

\section{Perturbation of the fluorescence spectrum at moderately high laser intensities $\left(\Gamma \ll \omega_{1}, \omega_{1}^{\prime} \ll s\right)$ : splitting of the Raman lines}

The method of calculation and the notation are the same as in I.

\subsection{Energy levels of the dressed atom: positions of the various components}

The laser frequency $\omega_{\mathrm{L}}$ is close to the frequency $\omega_{0}$ of the transition $e \rightarrow g$ :

where

$$
|\delta| \ll s
$$

$$
\delta=\omega_{0}-\omega_{\mathrm{L}}
$$

The laser intensity is sufficiently high to saturate the transition $e \rightarrow g\left(\omega_{1} \gg \Gamma\right.$, $\omega_{1} \geq \delta$ ) but not too high (the effect of the coupling between $e$ and $g^{\prime}$ remains weak $\left.\left(\omega_{1}^{\prime} \ll s\right)\right)$.

Note that the calculations presented in this section are also valid when the transition $e \rightarrow g$ is not saturated but the lines are well resolved $\left(\omega_{1}, \Gamma \ll \delta \ll a\right)$; in this case the conditions (3.1) of I are satisfied. This will permit a perturbative interpretation of the new lines obtained in this limit.

The unperturbed levels of the multiplicity $\mathscr{E}_{n}$ are shown in figure $3(a)$ where the broken arrows represent the matrix elements of the coupling $V$. As $\left|g^{\prime}, n+1\right\rangle$ is far from $|e, n\rangle\left(s \gg \omega_{1}^{\prime}\right)$, it is possible to consider first the effect of the coupling $\omega_{1}$. This leads to the energy diagram of figure $3(b),|\tilde{2, n}\rangle$ and $|\tilde{3, n}\rangle$ are linear combinations of $|e, n\rangle$ and $|g, n+1\rangle$

$$
\begin{aligned}
& |\tilde{2, n\rangle}=\cos \phi| e, n\rangle+\sin \phi|g, n+1\rangle \\
& |\tilde{3, n}\rangle=-\sin \phi|e, n\rangle+\cos \phi|g, n+1\rangle
\end{aligned}
$$




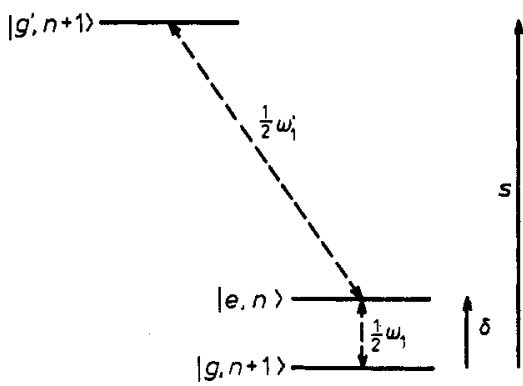

(a)

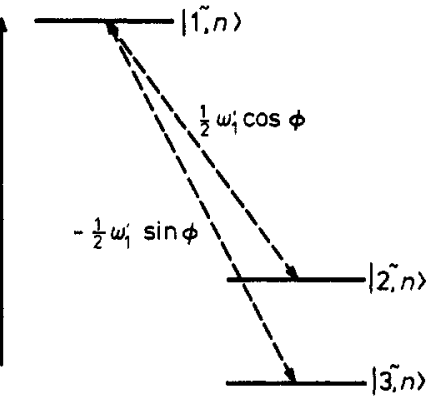

(b)

Figure 3. (a) Unperturbed levels of the multiplicity $\mathscr{E}_{n} . \delta$ is the laser detuning on the $e \rightarrow g$ transition. The coupling with the laser on the $e \rightarrow g, e \rightarrow g^{\prime}$ transitions (broken arrows) is characterized by the Rabi nutation frequencies $\omega_{1}, \omega_{1}^{\prime}$ which are supposed to be small compared to $s$. (b) Energy diagram obtained when the coupling $\omega_{1}$ with the $e \rightarrow g$ transition is diagonalized. The broken arrows represent the remaining coupling $\left(e \rightarrow g^{\prime}\right.$ transition). $\phi$ is defined by $\tan 2 \phi=\omega_{1} / \delta$.

with energies (with respect to $E_{g}=0$ )

$$
\begin{aligned}
& \tilde{E}_{2}=\left(\omega_{1}^{2}+\delta^{2}\right)^{1 / 2} \cos ^{2} \phi=\frac{1}{2}\left[\delta+\left(\omega_{1}^{2}+\delta^{2}\right)^{1 / 2}\right] \\
& \tilde{E}_{3}=-\left(\omega_{1}^{2}+\delta^{2}\right)^{1 / 2} \sin ^{2} \phi=\frac{1}{2}\left[\delta-\left(\omega_{1}^{2}+\delta^{2}\right)^{1 / 2}\right]
\end{aligned}
$$

where the parameter $\phi$ is defined by

$$
\tan 2 \phi=\omega_{1} / \delta
$$

$|\tilde{1, n}\rangle$ reduces to $\left|g^{\prime}, n+1\right\rangle$ (with energy $\tilde{E}_{1}=s$ ) and is coupled both to $\mid \tilde{2, n\rangle}$ and $|\tilde{3, n}\rangle$ (arrows in figure $3(b)$ ).

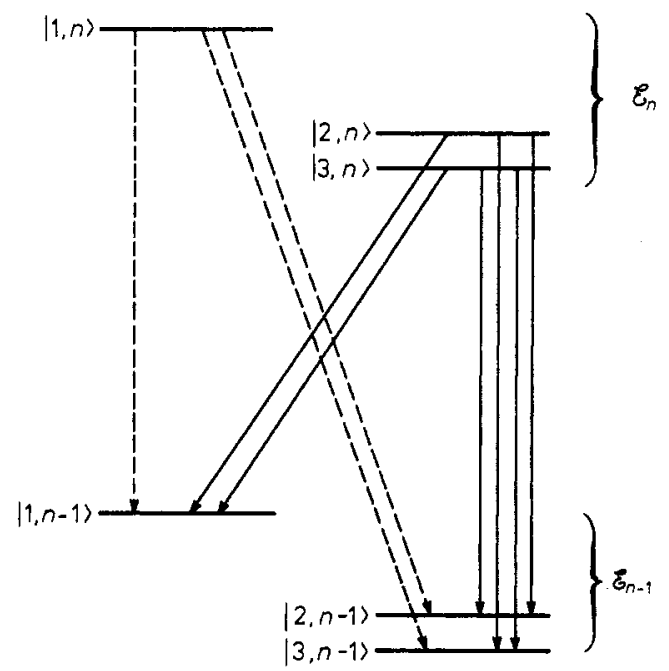

Figure 4. Multiplicities $\mathscr{E}_{n}$ and $\mathscr{E}_{n-1}$ of the dressed atom (perturbed energy levels). The arrows represent the allowed transitions (the broken arrows corresponding to transition rates vanishing if $\omega_{1}^{\prime} / s \rightarrow 0$ ). 
This coupling proportional to $\omega_{1}^{\prime}$ is now treated perturbatively. This leads to the perturbed energy diagram of figure 4 where the multiplicities $\mathscr{E}_{n}$ and $\mathscr{E}_{n-1}$ are represented. The level $|1, n\rangle$ contains a small admixture of $\mid \tilde{2, n\rangle}$ and $\mid \tilde{3, n\rangle}$, i.e. of $|e, n\rangle$ and $|g, n+1\rangle$, and is shifted upwards. Similar considerations apply to $|2, n\rangle$ and $|3, n\rangle$ (they are shifted downwards). Let us give the values of the splittings $\omega_{i j}$ between $|i, n\rangle$ and $|j, n\rangle$ calculated up to second order in $\omega_{1}^{\prime}$

$$
\begin{aligned}
& \omega_{12}=s-\frac{1}{2}\left[\delta+\left(\omega_{1}^{2}+\delta^{2}\right)^{1 / 2}\right]+\frac{\omega_{1}^{\prime 2}}{4 s}\left(1+\cos ^{2} \phi\right) \\
& \omega_{13}=s-\frac{1}{2}\left[\delta-\left(\omega_{1}^{2}+\delta^{2}\right)^{1 / 2}\right]+\frac{\omega_{1}^{\prime 2}}{4 s}\left(1+\sin ^{2} \phi\right) \\
& \omega_{23}=\left(\omega_{1}^{2}+\delta^{2}\right)^{1 / 2}-\frac{\omega_{1}^{\prime 2}}{4 s} \cos 2 \phi .
\end{aligned}
$$

The various corrections are all in $\omega_{1}^{\prime 2} / s$ and may be interpreted as resulting from light shifts of $g^{\prime}$ and $e$ due to the non-resonant excitation at frequency $\omega_{\mathrm{L}}$ of the transition $e \rightarrow g^{\prime}$.

The various components of the fluorescence spectrum at frequency $\omega_{\mathrm{L}}+\omega_{i j}$ correspond to the $|i, n\rangle \rightarrow|j, n-1\rangle$ allowed transitions represented by arrows on figure 4 .

The positions of the various components are represented on figure 5 in the case $\omega_{1} \ll \delta$, which allows a perturbative interpretation of the four new lines appearing at frequencies $\omega_{0}, 2 \omega_{\mathrm{L}}-\omega_{0}, \omega_{0}-s, 2 \omega_{\mathrm{L}}-\omega_{0}+s$ in addition to the three well known Rayleigh $\left(\omega_{\mathbf{L}}\right)$, Raman Stokes' $\left(\omega_{\mathbf{L}}-s\right)$ and Raman anti-Stokes' $\left(\omega_{\mathrm{L}}+s\right)$ lines. The corresponding non-linear scattering processes are sketched in figure 6 . Two laser photons $\omega_{\mathrm{L}}$ are absorbed and two fluorescence photons $\omega_{A}$ and $\omega_{B}$ are emitted. As the total energy is conserved during the transition, there is a strong correlation between the energies of the two fluorescence photons $\left(_{A}+\omega_{B}=2 \omega_{\mathrm{L}}\right.$ for figure $6(a) ; \omega_{A}+\omega_{B}=2 \omega_{\mathrm{L}}-s$ for figure $6(b) ; \omega_{A}+\omega_{B}=2 \omega_{\mathrm{L}}+s$ for figure $\left.6(c)\right)$. The transition amplitude is large when the energy of the third intermediate state coincides with the energy of $e$ (within the natural width $\gamma+\gamma^{\prime}$ of $e$ ). Although the sum of the energies of the two photons is well defined, each of these energies is distributed over an interval of the order of $\left(\gamma+\gamma^{\prime}\right)$. Finally the four new lines of figure 5 can be related to pairs of correlated fluorescence photons in non-linear scattering processes: $\left(\omega_{0}, 2 \omega_{\mathrm{L}}-\omega_{0}\right)$ for figure $6(a),\left(\omega_{0}-s, 2 \omega_{\mathrm{L}}-\omega_{0}\right)$ for figure $6(b)$ and

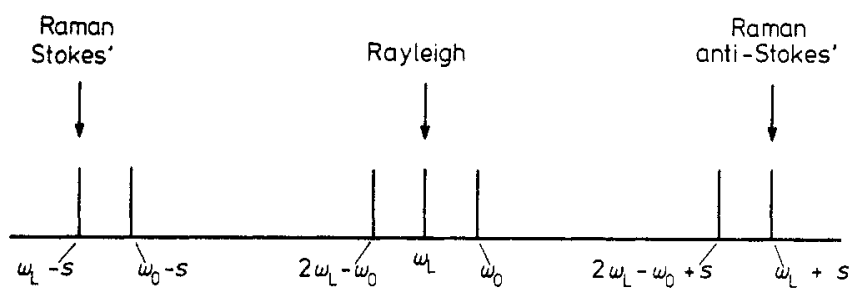

Figure 5. Position of the various components of the fluorescence spectrum for a non-zero detuning $\left(\delta=\omega_{0}-\omega_{\mathrm{L}} \neq 0\right)$ and at low intensities $\left(\omega_{1} \ll \delta\right)$. Four new lines appear in addition to the three lines corresponding to the diagrams of figure 2. Light shifts in $\omega_{1}^{2} / s$ have been neglected. 


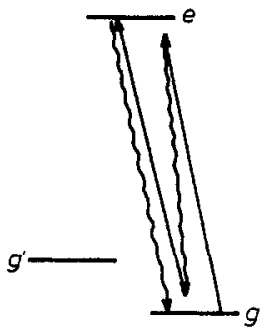

(a)

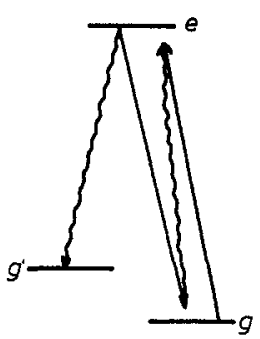

(b)

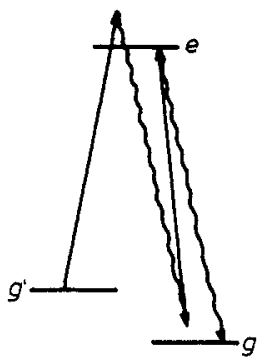

(c)

Figure 6. Non-linear scattering processes at the origin of the four new lines of figure 5. Two laser photons (full arrows) are absorbed and replaced by two fluorescence photons (wavy arrows) with frequencies (a) $\omega_{0}, 2 \omega_{\mathrm{L}}-\omega_{0}$, (b) $\omega_{0}-s, 2 \omega_{\mathrm{L}}-\omega_{0}$ and (c) $\omega_{0}, 2 \omega_{1}-\omega_{0}+s$.

$\left(\omega_{0}, 2 \omega_{\mathrm{L}}-\omega_{0}+s\right)$ for figure $6(c)$. Similar multiphoton processes are investigated in Mollow (1973).

At resonance $\omega_{\mathrm{L}}=\omega_{0}$, the spectrum also exhibits a triplet and two doublets (figure 7 ) but the splitting is now due to the Rabi nutation $\omega_{1}$ between $e$ and $g$ and not to the laser detuning $\delta$ (expressions (3.6)). Since we suppose $\omega_{1} \gg \Gamma$, several nutations occur between $e$ and $g$ during the radiative lifetime, which excludes any perturbative approach of the type of figure 6. The central triplet and the two lateral doublets are the manifestations of the dynamical resonant Stark splitting on the Rayleigh and Raman (Stokes' and anti-Stokes') lines. Corrections in $\omega_{1}^{\prime 2}$ to the various splittings are given in expressions (3.6).

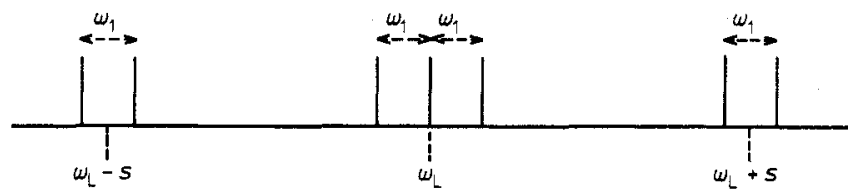

Figure 7. Positions of the various components of the fluorescence spectrum at resonance $\left(\omega_{\mathrm{L}}=\omega_{0}\right)$ at intermediate intensities $\left(\Gamma \ll \omega_{1} \ll s\right)$. Light shifts in $\omega_{1}^{\prime 2} / s$ have been neglected. The dynamical Stark splitting appears on Rayleigh and Raman lines.

\subsection{Transition rates: widths of the lateral components}

From the perturbative expression (in $\omega_{1}^{\prime} / s$ ) of the $|i, n\rangle$ states, one can easily calculate the matrix elements of $D$

$$
\boldsymbol{d}_{j i}^{-}=\langle j, n-1|\boldsymbol{D}| i, n\rangle
$$

and the corresponding total spontaneous transition rates

$$
\Gamma_{j i}=\left|\boldsymbol{d}_{j i}^{-}\right|^{2} .
$$

A straightforward calculation leads to the results of table 1.

Each transition rate $\Gamma_{j i}$ is calculated at the lowest order, and, at this order, is found to be proportional either to $\gamma$ or to $\gamma^{\prime}$, the fluorescence photons emitted from this transition having a well defined polarization $\hat{\boldsymbol{\epsilon}}$ or $\hat{\boldsymbol{\epsilon}}^{\prime}$ (this would not be true at higher orders). The transitions for which $\Gamma_{j i}$ vanishes when $\omega_{1}^{\prime}$ tends to zero, 
Table 1.

\begin{tabular}{llll}
\hline$j$ & 2 & 3 \\
\hline 1 & $\gamma^{\prime} \omega_{1}^{\prime 2} / 4 s^{2}$ & & \\
2 & $\left(\gamma \omega_{1}^{\prime 2} / 4 s^{2}\right) \sin ^{2} \phi$ & $\gamma^{\prime} \cos ^{2} \phi$ & $\gamma \sin ^{2} \phi$ \\
3 & $\left(\gamma \omega_{1}^{\prime 2} / 4 s^{2}\right) \cos ^{2} \phi$ & $\gamma \sin ^{2} \phi \cos ^{2} \phi$ & $\gamma \sin ^{4} \phi$ \\
\hline
\end{tabular}

are represented by broken arrows on figure 4 (they all start from $|1, n\rangle$ and only exist because of the contamination of $\left|g^{\prime}, n+1\right\rangle$ by $\left.|e, n\rangle\right)$.

From expression (4.19) of I one easily calculates the widths $L_{i j}$ of the lateral components at frequency $\omega_{\mathrm{L}}+\omega_{i j}$

$$
L_{i j}=\frac{1}{2}\left(\Gamma_{i}+\Gamma_{j}\right)-\boldsymbol{d}_{i i}^{+} \cdot \boldsymbol{d}_{j j}^{-}
$$

where

$$
\Gamma_{i}=\sum_{l} \Gamma_{l i} .
$$

Let us recall that the last term of (3.9) describes the effect of cascades. One finds:

$$
\begin{aligned}
& L_{23}=L_{32}=\frac{1}{2}\left(\gamma+\gamma^{\prime}\right)+\gamma \sin ^{2} \phi \cos ^{2} \phi \\
& L_{21}=L_{12}=\frac{1}{2}\left(\gamma+\gamma^{\prime}\right) \cos ^{2} \phi \\
& L_{31}=L_{13}=\frac{1}{2}\left(\gamma+\gamma^{\prime}\right) \sin ^{2} \phi .
\end{aligned}
$$

It may be surprising that the Raman Stokes' and anti-Stokes' lines have a finite width. This is due to the fact that, at high intensities $\left(\omega_{1} \gg \Gamma\right)$ the instability of $e$ is shared between $e$ and $g$, which gives a finite width to the initial or final state of the Raman processes.

The values of the $L_{i j}$ (and the weights of the components given in (3.15) \$3.3) at the limit $\delta \gg \omega_{1}$, are in agreement with the perturbative predictions of the previous section: one gets three narrow lines at $\omega_{\mathrm{L}}, \omega_{\mathrm{L}}-s, \omega_{\mathrm{L}}+s$ and four broad (width $\left.\simeq \frac{1}{2}\left(\gamma+\gamma^{\prime}\right)\right)$ and weaker (by a factor $\left.\omega_{1}^{2} / 4 \delta^{2}\right)$ lines at $\omega_{0}, 2 \omega_{\mathrm{L}}-\omega_{0}, \omega_{0}-s$, $2 \omega_{\mathrm{L}}-\omega_{0}+s$.

\subsection{Evolution of the populations: weights of the components}

In $\mathrm{I}$, it is shown that the populations of the energy level $|i, n\rangle$ of the dressed atom can be written

$$
\sigma_{i i n}(t)=\Pi_{i}(t) p_{0}(n)
$$

where $p_{0}(n)$ is the normalized distribution of the number $n$ of photons in the laser mode and where the $\Pi_{i}(t)$ satisfy the rate equations

$$
\dot{\Pi}_{i}(t)=-\left(\sum_{j \neq i} \Gamma_{j i}\right) \Pi_{i}(t)+\sum_{i \neq i} \Gamma_{i l} \Pi_{l}(t) .
$$

We suppose that the atom is in $g$ at $t=0$.

The knowledge of the $\Pi_{i}(t)$ is important for the computation of the weights of the different components. Simple expressions with an obvious physical meaning are 
derived in I for the weights $\mathscr{J}\left(\omega_{\mathrm{L}}+\omega_{i j}\right), \mathscr{J}\left(\omega_{\mathrm{L}}\right)$ of the lateral and central components

$$
\begin{aligned}
& \mathscr{J}\left(\omega_{\mathbf{L}}+\omega_{i j}\right)=\Gamma_{j i} \int_{0}^{T} \Pi_{i}(t) \mathrm{d} t \\
& \mathscr{J}\left(\omega_{\mathrm{L}}\right)=\sum_{i} \Gamma_{i i} \int_{0}^{T} \Pi_{i}(t) \mathrm{d} t .
\end{aligned}
$$

Equations (3.13) $(i=1,2,3)$ are solved in the appendix. A Laplace transformation allows a simple evaluation of the contribution to $\int \Pi_{i}(t)$ of the transient and steady-state regimes (in the case of a square variation over an interval $T$ of the laser intensity experienced by the atom). One can show that the two time constants associated with the transient regime, and corresponding to the $\Gamma_{i j}$ given in table 1 , are of the order of $\left(\gamma+\gamma^{\prime}\right)^{-1}$ (provided that $\delta$ is not too large compared to $\omega_{1}$ ). This means that the transient regime lasts a short time and can be suppressed by not observing the light emitted at the edges of the illuminated portion of the atomic beam.

Equations (3.15) give the weights $\mathscr{J}\left(\omega_{\mathrm{L}}+\omega_{i i}\right), \mathscr{J}\left(\omega_{\mathrm{L}}\right)$ computed from (3.14) and the results of the appendix

$$
\begin{aligned}
& \mathscr{J}\left(\omega_{\mathrm{L}}\right)=\frac{\gamma}{\gamma^{\prime}}\left(\sin ^{4} \phi+\cos ^{4} \phi\right)\left(1+\gamma T \frac{\omega_{1}^{\prime 2}}{4 s^{2}}\right)+\gamma^{\prime} T \frac{\omega_{1}^{\prime 2}}{4 s^{2}} \\
& \mathscr{J}\left(\omega_{\mathrm{L}}+\omega_{23}\right)=\mathscr{J}\left(\omega_{\mathrm{L}}+\omega_{32}\right)=\frac{\gamma}{\gamma^{\prime}} \sin ^{2} \phi \cos ^{2} \phi\left(1+\gamma T \frac{\omega_{1}^{\prime 2}}{4 s^{2}}\right) \\
& \mathscr{J}\left(\omega_{\mathrm{L}}+\omega_{21}\right)=\sin ^{2} \phi\left(1+\gamma T \frac{\omega_{1}^{\prime 2}}{4 s^{2}}\right) \\
& \mathscr{J}\left(\omega_{\mathrm{L}}+\omega_{31}\right)=\cos ^{2} \phi\left(1+\gamma T \frac{\omega_{1}^{\prime 2}}{4 s^{2}}\right) \\
& \mathscr{J}\left(\omega_{\mathrm{L}}+\omega_{12}\right)=\sin ^{2} \phi \gamma T \frac{\omega_{1}^{\prime 2}}{4 s^{2}} \\
& \mathscr{J}\left(\omega_{\mathrm{L}}+\omega_{13}\right)=\cos ^{2} \phi \gamma T \frac{\omega_{1}^{\prime 2}}{4 s^{2}} .
\end{aligned}
$$

The terms proportional to the transit time $T$ represent the contribution of the steady-state regime, those independent of $T$ come from the transient one. The transitions ending at $|1, n\rangle$ have an $\hat{\boldsymbol{\epsilon}}^{\prime}$ polarization, the others have an $\hat{\boldsymbol{\epsilon}}$ polarization.

All the contributions of the steady-state regime are proportional to $\omega_{1}^{\prime 2} / 4 s^{2}$. This can be easily understood. If $\omega_{1}^{\prime}=0$, an atom which has been resonantly excited from $g$ to $e$ and which falls back by spontaneous emission in $g^{\prime}$ is trapped and definitely lost. The steady-state regime is, in this case, $\Pi_{1}=1, \Pi_{2}=\Pi_{3}=0$, and no fluorescence light is emitted. When $\omega_{1}^{\prime} \neq 0$ (in this section, we suppose $\omega_{1}^{\prime} \ll s$ ), the trapping in $g^{\prime}$ is not perfect since an atom in $g^{\prime}$ has a weak probability of being re-excited in $e$. In the dressed atom picture, this corresponds to the small contamination of $\left|g^{\prime}, n+1\right\rangle$ by $|e, n\rangle$, which allows weak transition rates $\Gamma_{21}, \Gamma_{31}$ between $|1, n\rangle$ and $|2, n-1\rangle,|3, n-1\rangle$ (broken arrows on figure 4) of the order of $\gamma \omega_{1}^{\prime 2} / 4 s^{2}$. The steady-state regime in this case corresponds to values of $\Pi_{2}$ and $\Pi_{3}$ of the 
order of $\omega_{1}^{\prime 2} / 4 s^{2}$ while $\Pi_{1}$ remains of the order of one. The transitions starting from $|1, n\rangle$ have a weak transition rate, but they are proportional to $\Pi_{1}$, which is large. This explains why they have a weight comparable to that of other transitions.

As a consequence of the smallness of the steady-state contribution (in $\omega_{1}^{\prime 2} / 4 s^{2}$ ), one must keep the transient one. Since, in the steady state $\Pi_{2}, \Pi_{3} \ll \Pi_{1} \simeq 1$, the transient contribution affects essentially the components starting from $|2, n\rangle|3, n\rangle$ (see equations (3.15)). This leads to asymmetries in the spectrum, even at resonance (see discussion in I \$5.1). The anti-Stokes' doublet is weaker than the Stokes' one, the difference corresponding to the light emitted during the transient regime from the initial state where all atoms are in $g$.

Finally, the intensity of the elastic component at $\omega_{\mathrm{L}}$ can be calculated from expression (5.24) of I. One finds

$$
\mathscr{F}_{\mathrm{el}}=\Gamma_{11} \Pi_{1}(+\infty) T=\gamma^{\prime} T \omega_{1}^{\prime 2} / 4 s^{2} .
$$

Such a result is simple to understand: most atoms are in $g^{\prime}$; the elastic component corresponds to the Rayleigh non-resonant scattering on $e \rightarrow g^{\prime}$.

\section{Perturbation of the fluorescence spectrum at very high intensities $\left(\omega_{1} \gg s \gg \Gamma\right)$ : complete mixing between Rayleigh and Raman lines}

\subsection{Positions of the various components}

The laser intensity is now so large that the splitting $s$ between $g$ and $g^{\prime}$ becomes much smaller than the Rabi nutation frequencies $\omega_{1}, \omega_{1}^{\prime}\left(\omega_{1}, \omega_{1}^{\prime} \gg s\right)$. We suppose in addition that the laser detuning $\delta=\omega_{0}-\omega_{\mathrm{L}}$ is zero, which implies that both transitions $e \rightarrow g$ can be considered as resonant.

In such a situation, it seems better to diagonalize the total coupling $V$ with the laser, neglecting in the first step $s$, and then to treat perturbatively the effect of the structure.

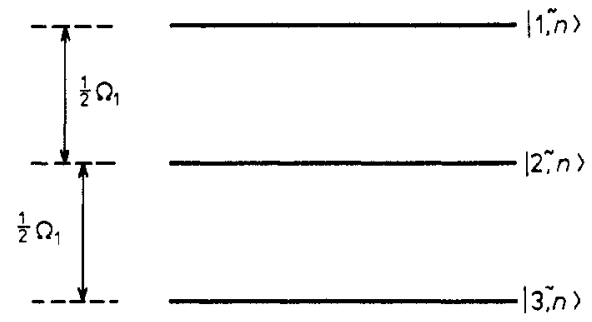

Figure 8. Energy levels $|i, \tilde{n}\rangle(i=1,2,3)$ of the multiplicity $\mathscr{E}_{n}$ of the dressed atom when the splitting $s$ is neglected in comparison with $\Omega_{1}=\left(\omega_{1}^{2}+\omega_{1}^{\prime 2}\right)^{1 / 2}$ (very high intensity limit). The detuning $\delta=\omega_{0}-\omega_{\mathrm{L}}$ is zero so that the three levels are equally spaced.

The eigenstates and eigenvalues of $V$ are easily found (see figure 8)

$$
\begin{aligned}
|\tilde{1, n}\rangle & =\frac{1}{\sqrt{ } 2}(|e, n\rangle+|G, n+1\rangle) & & \tilde{E_{1}}=\frac{1}{2} \Omega_{1} \\
|\tilde{2, n}\rangle & =\left|G^{\prime}, n+1\right\rangle & & \tilde{E_{2}}=0 \\
|\tilde{3, n}\rangle & =\frac{1}{\sqrt{2}}(|e, n\rangle-|G, n+1\rangle) & \tilde{E_{3}} & =-\frac{1}{2} \Omega_{1}
\end{aligned}
$$


where

$$
\begin{aligned}
& \Omega_{1}=\left(\omega_{1}^{2}+\omega_{1}^{\prime 2}\right)^{1 / 2} \\
& |G, n+1\rangle=\frac{1}{\Omega_{1}}\left(\omega_{1}|g, n+1\rangle+\omega_{1}^{\prime}\left|g^{\prime}, n+1\right\rangle\right) \\
& \left|G^{\prime}, n+1\right\rangle=\frac{1}{\Omega_{1}}\left(\omega_{1}^{\prime}|g, n+1\rangle-\omega_{1}\left|g^{\prime}, n+1\right\rangle\right) .
\end{aligned}
$$

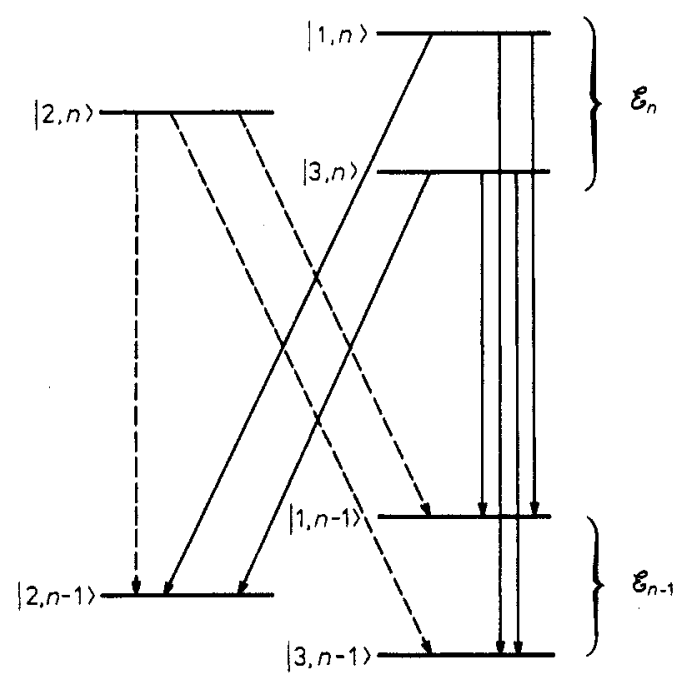

Figure 9. Perturbed energy diagram derived from the one of figure 8 , when the non-zero value of $s$ is taken into account. Two multiplicities $\mathscr{E}_{n}$ and $\mathscr{E}_{n-1}$ are represented with allowed transitions between them, broken arrows corresponding to transitions vanishing if $s / \Omega_{1} \rightarrow 0$.

The remaining part of the dressed-atom Hamiltonian $H-V$ is now treated perturbatively, introducing a displacement and a contamination of each level $|\tilde{1, n}\rangle$. This leads to the perturbed energy diagram of figure 9. Straightforward perturbative calculations give for the spacings $\omega_{i j}$ between $|i, n\rangle$ and $|j, n\rangle$

$$
\begin{aligned}
& \omega_{12}=\frac{\Omega_{1}}{2}+\frac{s}{2} \frac{\omega_{1}^{\prime 2}-2 \omega_{1}^{2}}{\Omega_{1}^{2}}+\frac{s^{2} \omega_{1}^{\prime 2}\left(\omega_{1}^{\prime 2}+4 \omega_{1}^{2}\right)}{4 \Omega_{1}^{5}} \\
& \omega_{23}=\frac{\Omega_{1}}{2}-\frac{s}{2} \frac{\omega_{1}^{\prime 2}-2 \omega_{1}^{2}}{\Omega_{1}^{2}}+\frac{s^{2} \omega_{1}^{\prime 2}\left(\omega_{1}^{\prime 2}+4 \omega_{1}^{2}\right)}{4 \Omega_{1}^{5}} \\
& \omega_{13}=\Omega_{1}+\frac{s^{2} \omega_{1}^{\prime 2}\left(\omega_{1}^{\prime 2}+4 \omega_{1}^{2}\right)}{2 \Omega_{1}^{5}}
\end{aligned}
$$

and consequently the positions $\omega_{\mathrm{L}}+\omega_{i j}$ of the various components of the fluorescence spectrum (see figure 10). This spectrum exhibits five groups of lines around 


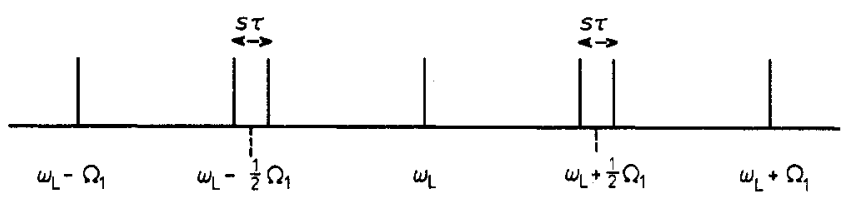

Figure 10. Positions of the various components of the fluorescence spectrum in the very high intensity limit $\left(s \ll \Omega_{1}\right.$ ) and corresponding to the diagram of figure 9 . The structure is completely different from the one of figure 7. Five groups of lines separated by $\frac{1}{2} \Omega_{1}$ appear. The splitting of the two doublets is proportional to the structure $s$ of the bare atom $\left(\tau=\left(\omega_{1}^{\prime 2}-2 \omega_{1}^{2}\right) / \Omega_{1}^{2}\right)$.

$\omega_{\mathrm{L}}, \omega_{\mathrm{L}} \pm \frac{1}{2} \Omega_{1}, \omega_{\mathrm{L}} \pm \Omega_{1}$ with two doublets around $\omega_{\mathrm{L}} \pm \frac{1}{2} \Omega_{1}$ with a splitting of the order of $s$

$$
\omega_{12}-\omega_{23}=s \tau
$$

where

$$
\tau=\frac{\omega_{1}^{\prime 2}-2 \omega_{1}^{2}}{\omega_{1}^{\prime 2}+\omega_{1}^{2}} .
$$

This spectrum is quite different from that of figure 7. It is interesting to study the transition between these two extremes. This can be done (Reynaud 1977) by diagonalizing the $3 \times 3$ matrix representing the dressed-atom Hamiltonian $H$ in the multiplicity $\left\{|e, n\rangle,|g, n+1\rangle,\left|g^{\prime}, n+1\right\rangle\right\}$. This diagonalization gives the $\omega_{i j}$ for any value of $\omega_{1}$. The results are given in figure 11 in the case $\omega_{1}=\omega_{1}^{\prime}, \omega_{\mathbf{L}}=\omega_{0}$. Various spectra corresponding to different values of $\omega_{1} / \mathrm{s}$ are represented, the broken lines

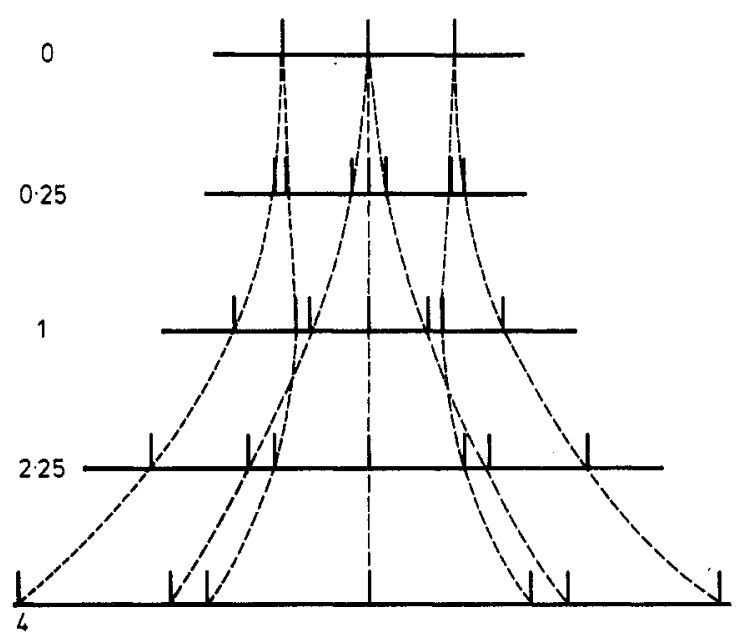

Figure 11. Evolution of the positions of the various components of the fluorescence spectrum when the light intensity is progressively increased from very low to very high values. Each spectrum corresponds to a value of the dimensionless parameter $\Omega_{1} / \mathrm{s}$ indicated on the figure. The broken lines represent the continuous evolution of a given component. 
showing the continuous evolution of a given linet. The separation between the two outer components of the Stokes' and anti-Stokes' doublets starts from $2 s$ and increases as $\omega_{1}$ for $\omega_{1} \ll s$, as $\left(\omega_{1}^{2}+\omega_{1}^{\prime 2}\right)^{1 / 2}=\sqrt{2} \omega_{1}$ for $\omega_{1} \gg s$. The two inner components of the Stokes' and anti-Stokes' doublets join the two lateral components of the Rayleigh triplet to form the two doublets of figure 10. This shows the complete mixing between Raman and Rayleigh lines at high intensities.

\subsection{Widths of the various components}

To simplify the notation and make the discussion clearer, let us introduce

$$
\begin{array}{ll}
\langle G|\boldsymbol{D}| e\rangle=d_{G} \hat{\boldsymbol{\epsilon}}_{G} & \Gamma_{G}=\left|d_{G}\right|^{2} \\
\left\langle G^{\prime}|\boldsymbol{D}| e\right\rangle=d_{G^{\prime}} \hat{\boldsymbol{\epsilon}}_{G^{\prime}} & \Gamma_{G^{\prime}}=\left|d_{G^{\prime}}\right|^{2} .
\end{array}
$$

One can compute the polarizations $\hat{\boldsymbol{\epsilon}}_{G}, \hat{\boldsymbol{\epsilon}}_{G^{\prime}}$ and the transition rates $\Gamma_{G}, \Gamma_{G^{\prime}}$ from the expressions for $G$ and $G^{\prime}$ (see (4.3)) and from the definition of $D$ in the bare atom basis (see (2.1) and (2.2)):

$$
\begin{array}{ll}
\Gamma_{G}=\frac{\gamma \omega_{1}^{2}+\gamma^{\prime} \omega_{1}^{\prime 2}}{\Omega_{1}^{2}} & \hat{\boldsymbol{\epsilon}}_{G}=\frac{d \omega_{1} \hat{\boldsymbol{\epsilon}}+d^{\prime} \omega_{1}^{\prime} \hat{\boldsymbol{\epsilon}}^{\prime}}{\left(\gamma \omega_{1}^{2}+\gamma^{\prime} \omega_{1}^{\prime 2}\right)^{1 / 2}} \\
\Gamma_{G^{\prime}}=\frac{\gamma \omega_{1}^{\prime 2}+\gamma^{\prime} \omega_{1}^{2}}{\Omega_{1}^{2}} & \hat{\boldsymbol{\epsilon}}_{G^{\prime}}=\frac{d \omega_{1}^{\prime} \hat{\boldsymbol{\epsilon}}-d^{\prime} \omega_{1} \hat{\boldsymbol{\epsilon}}^{\prime}}{\left(\gamma \omega_{1}^{\prime 2}+\gamma^{\prime} \omega_{1}^{2}\right)^{1 / 2}} .
\end{array}
$$

The two polarizations $\hat{\boldsymbol{\epsilon}}_{G}$ and $\hat{\boldsymbol{\epsilon}}_{G^{\prime}}$ are not in general orthogonal since

$$
\langle G|\boldsymbol{D}| e\rangle\left\langle e|\boldsymbol{D}| G^{\prime}\right\rangle=\left(\gamma-\gamma^{\prime}\right) \frac{\omega_{1} \omega_{1}^{\prime}}{\Omega_{1}^{2}} .
$$

The polarization $\hat{\boldsymbol{\epsilon}}_{G^{\prime}}$ is orthogonal to the laser beam polarization since $\left|G^{\prime}\right\rangle$ is defined as uncoupled to the level $|e\rangle$ by the laser-atom interaction Hamiltonian $\left(\hat{\epsilon}_{\mathrm{L}} \cdot D^{+}+\hat{\epsilon}_{\mathrm{L}}^{*} \cdot D^{-}\right)$.

The perturbed eigenstates are easily calculated and as in (3.2), it is possible to compute the matrix elements $\boldsymbol{d}_{j i}^{-}$of the atomic dipole moment and the transition rates $\Gamma_{j i}$. One gets the results of table 2 , where $k=4 \omega_{1}^{2} \omega_{1}^{\prime 2} / \Omega_{1}^{4}$.

Each $\Gamma_{j i}$ (which are here calculated in the lowest order) is proportional to $\Gamma_{G}$ or $\Gamma_{G^{\prime}}$ and the fluorescence photons have the corresponding polarization $\hat{\boldsymbol{\epsilon}}_{G}$ or $\hat{\boldsymbol{\epsilon}}_{G^{\prime}}$ : $\hat{\boldsymbol{\epsilon}}_{G^{\prime}}$ for the transitions ending at $|2, n-1\rangle$ since $|2, n-1\rangle$ is essentially $\left|G^{\prime}, n\right\rangle$

Table 2.

\begin{tabular}{llll}
\hline$j \swarrow i$ & 1 & 3 \\
\hline 1 & $\frac{1}{4} \Gamma_{G}$ & $\Gamma_{G} k s^{2} / 2 \Omega_{1}^{2}$ & \\
2 & $\frac{1}{2} \Gamma_{G^{\prime}}$ & $\Gamma_{G^{\prime}} k s^{2} / \Omega_{1}^{2}$ & $\frac{1}{4} \Gamma_{G}$ \\
3 & $\frac{1}{4} \Gamma_{G}$ & $\Gamma_{G} k s^{2} / 2 \Omega_{1}^{2}$ & $\frac{1}{2} \Gamma_{G^{\prime}}$ \\
\hline
\end{tabular}

$\uparrow$ The indices 1, 2, 3 labelling the three eigenstates $|i, n\rangle$ of $H$ have been chosen in such a way that the level labelled $|i, n\rangle$ at low intensity (in $\$ 3$ ) becomes by continuity the level $|i, n\rangle$ at high intensity (in \$4). 
and $\hat{\boldsymbol{\epsilon}}_{G}$ for the other transitions since $|1, n-1\rangle$ and $|3, n-1\rangle$ contain much more $|G, n\rangle$ than $\left|G^{\prime}, n\right\rangle$. As a consequence of the complete mixing between $g$ and $g^{\prime}$, there is a complete mixing between the polarizations $\hat{\boldsymbol{\epsilon}}$ and $\hat{\boldsymbol{\epsilon}}^{\prime}$.

From the general expression (3.9) for the width $L_{i j}$ of the lateral components, one derives in the lowest order

$$
\begin{aligned}
& L_{12}=L_{21}=L_{23}=L_{32}=\frac{1}{4}\left(\Gamma_{G}+\Gamma_{G^{\prime}}\right)=\frac{1}{4}\left(\gamma+\gamma^{\prime}\right) \\
& L_{13}=L_{31}=\frac{3}{4} \Gamma_{G}+\frac{1}{2} \Gamma_{G^{\prime}} .
\end{aligned}
$$

\subsection{Weights of the components}

A calculation analogous to the one of $\$ 3.3$ and based on expression (3.14) and on the results of the appendix leads to the following predictions for the weights of the components observed without the polarization analyser:

$$
\begin{aligned}
& \left.\mathscr{J}\left(\omega_{\mathrm{L}}\right)=\frac{\Gamma_{G}}{2 \Gamma_{G^{\prime}}}\left(\frac{\omega_{1}^{2}}{\Omega_{1}^{2}}+\Gamma_{G} T k \frac{s^{2}}{\Omega_{1}^{2}}\right)+\Gamma_{G^{\prime}} T k \frac{s^{2}}{\Omega_{1}^{2}}\right) \\
& \mathscr{J}\left(\omega_{\mathrm{L}}+\omega_{13}\right)=\mathscr{J}\left(\omega_{\mathrm{L}}+\omega_{31}\right)=\frac{\Gamma_{G}}{4 \Gamma_{G^{\prime}}}\left(\frac{\omega_{1}^{2}}{\Omega_{1}^{2}}+\Gamma_{G} T k \frac{s^{2}}{\Omega_{1}^{2}}\right) \\
& \mathscr{J}\left(\omega_{\mathrm{L}}+\omega_{12}\right)=\mathscr{J}\left(\omega_{\mathrm{L}}+\omega_{32}\right)=\frac{1}{2}\left(\frac{\omega_{1}^{2}}{\Omega_{1}^{2}}+\Gamma_{G} T k \frac{s^{2}}{\Omega_{1}^{2}}\right) \\
& \mathscr{J}\left(\omega_{\mathrm{L}}+\omega_{21}\right)=\mathscr{J}\left(\omega_{\mathrm{L}}+\omega_{23}\right)=\frac{\Gamma_{G}}{2} T k \frac{s^{2}}{\Omega_{1}^{2}} .
\end{aligned}
$$

As in $\$ 3.3$, the terms proportional to $T$ represent the steady-state contribution. The fluorescence photons have an $\hat{\boldsymbol{\epsilon}}_{G^{\prime}}$ polarization for the transitions ending in $|2, n-1\rangle$ and an $\hat{\boldsymbol{\epsilon}}_{G}$ polarization for the other ones.

All the contributions of the steady-state regime decrease with the laser intensity as $s^{2} / \Omega_{1}^{2}$. Compared with the results of $\S 3.3$, one finds that the steady-state contribution first increases as $\omega_{1}^{\prime 2} / s^{2}$ at low intensity $\left(\omega_{1}^{\prime} \ll s\right)$ and then decreases as $s^{2} / \Omega_{1}^{2}$ at high intensity $\left(s \ll \Omega_{1}\right)$ which means that a maximum of fluorescence is expected around $\omega_{1}, \omega_{1}^{\prime} \simeq s$. The decrease of fluorescence at high intensity can be understood in the following way. Suppose first that $s=0$. As $g$ and $g^{\prime}$ are degenerate, the two linear combinations $G$ and $G^{\prime}$ introduced in (4.3) are also eigenstates of the (bare) atomic Hamiltonian $H_{0}$. One of them, $G$, is coupled by the laser to $e$, whereas the second is not (the quantities $\Gamma_{G}$ and $\Gamma_{G^{\prime}}$ introduced in (4.6) are just the spontaneous transition rates $e \rightarrow G$ and $e \rightarrow G^{\prime}$ ). An atom excited from $G$ to $e$ and falling back to $G^{\prime}$ is trapped in $G^{\prime}$ and definitely lost. In the dressed-atom picture, the level $|2, n\rangle$ reduced to $\left|G^{\prime}, n+1\right\rangle$ and is not coupled by spontaneous emission to any lower level. A small splitting $s$ couples $|2, n\rangle$ to $|1, n\rangle$ and $|3, n\rangle$ and introduces in $|2, n\rangle$ a small admixture of $|e, n\rangle$ (of the order of $s / \Omega_{1}$ ); atoms trapped in $\left|G^{\prime}, n+1\right\rangle$ have now a small probability (of the order of $s^{2} / \Omega_{1}^{2}$ ) of falling back to $|1, n\rangle$ or $|3, n\rangle$ (broken arrows on figure 9). In the steady-state regime, $\Pi_{1}$ and $\Pi_{3}$ are of the order of $s^{2} / \Omega_{1}^{2}$ and $\Pi_{2}$ is of the order of one. Although the physical origin of the trap level is not the same, the situation is quite similar to the one 
of $\$ 3.3$ and the discussion is the same (importance of the transient regime, asymmetries, elastic component equal to $\left.\Gamma_{22} \Pi_{2}(+\infty) T, \ldots\right)$.

\subsection{Preparation of a static coherent superposition of the lower sublevels}

It should be noted that high-intensity laser irradiation $\left(\omega_{1}, \omega_{1}^{\prime} \gg s\right)$ produces atoms in a coherent superposition of $g$ and $g^{\prime}$. As mentioned above, in the steady-state regime, $\Pi_{2}$ is much larger than $\Pi_{1}$ and $\Pi_{3}$, which means that the level $|2, n\rangle$ which is essentially $\left|G^{\prime}, n+1\right\rangle$ is the most populated. To understand the building up of such a coherence between $g$ and $g^{\prime}$, let us start with a statistical mixture in equal proportion of $g$ and $g^{\prime}$, i.e. also $G$ and $G^{\prime}$. Atoms in $G$ oscillate between $G$ and $e$ at frequency $\Omega_{1}$ under the effect of the laser irradiation. Some of them fall back to $G^{\prime}$ which is not coupled to $e$. The atomic structure $s$ introduces an oscillation at frequency $s$ between $G$ and $G^{\prime}$, but, as $s \ll \Omega_{1}$, this oscillation cannot remove the excess of atoms accumulated in $G^{\prime}$. When the atoms leave the laser beam, such a coherence between $g$ and $g^{\prime}$ which was static in the presence of the laser irradiation oscillates freely at frequency $s$ and without any damping since $g$ and $g^{\prime}$ are groundstate sublevels and since collisions are completely negligible in an atomic beam. This coherence could be detected by monitoring the modulation of the absorption of a weak probe light beam.

Finally let us mention that similar effects are expected when the structure exists in the upper state instead of the lower one. In this case, there is also a linear combination $E$ of $e$ and $e^{\prime}$ which is coupled to $g$ by the laser while the other, $E^{\prime}$, is not. It may be shown that, at very high intensities, $E^{\prime}$ plays a negligible role in this problem so that we are led to a two-level problem $(g, E)$. Half of the atoms are in $E$, i.e. in a well defined static coherent superposition of $e$ and $e^{\prime}$. When atoms leave the laser beam (and if they leave it abruptly), such a coherence could be detected by a modulation of the fluorescence light at the frequency determined by the structure $e-e^{\prime}$ (quantum beats).

\section{Absorption of a weak probe light beam}

In addition to the strong laser light which saturates the three-level atom, we now consider a second weak probe beam irradiating the atomic beam at right angles.

It is shown in I that the components of the absorption spectrum observed on such a probe beam have the same positions and widths as those of the fluorescence spectrum and simple expressions are derived for their intensities:

$$
\mathscr{J}_{A}\left(\omega_{\mathrm{L}}+\omega_{i j}\right)=K \Gamma_{j i} \int_{0}^{T}\left(\Pi_{j}(t)-\Pi_{i}(t)\right) \mathrm{d} t
$$

where $K$ is proportional to the intensity of the probe laser beam and to the optical depth of the atomic beam.

The intensity of an absorption line is the difference between pure absorption processes and stimulated emission processes. The absorption and stimulated emission transition rates are equal and the intensity is therefore proportional to the difference of populations of the lower and upper states. This explains why the central component disappears in the absorption spectrum. 
If the probe laser beam has a well defined polarization $\hat{\boldsymbol{\epsilon}}_{\mathrm{D}}$, the expression (5.1) is modified by changing $\Gamma_{j i}=\left|\boldsymbol{d}_{j i}^{-}\right|^{2}$ into $\Gamma_{j i}\left(\hat{\boldsymbol{\epsilon}}_{\mathrm{D}}\right)=\left|\boldsymbol{d}_{j i}^{-}, \hat{\boldsymbol{\epsilon}}_{\mathrm{D}}\right|^{2}$ (as for the fluorescence spectrum when the fluorescence photons are detected with a given polarization $\hat{\boldsymbol{\epsilon}}_{\mathrm{D}}$ ).

Let us consider first the case of moderately high intensities of the (saturating) laser light $\left(\Gamma \ll \omega_{1}, \omega_{1}^{\prime} \ll s\right)$. From the results of $\S 3$ and from (5.1), one easily shows that the absorption spectrum observed on the probe beam consists essentially of two absorbing Raman Stokes' lines. These transitions correspond indeed to non-weak transition rates (full arrows on figure 4) and to a non-weak value of the population of the lower state $\left(\Pi_{1} \simeq 1\right)$. The Raman anti-Stokes' lines are amplifying lines weaker than the Raman Stokes' ones by a factor of the order of $\omega_{1}^{\prime 2} / 4 s^{2}$, since the corresponding transition rates are of the order of $\gamma \omega_{1}^{\prime 2} / 4 s^{2}$. Of the two Rayleigh lateral lines, one is amplifying, the other is absorbing, and they are also weaker than the Raman Stokes' ones by a factor of the order $\omega_{1}^{\prime 2} / 4 s^{2}$ because of the weakness of the populations of both upper and lower states.

To summarize, the absorption spectrum consists mainly of two absorbing lines around $\omega_{\mathrm{L}}-s$ with a splitting $\left(\omega_{1}^{2}+\delta^{2}\right)^{1 / 2}$, and there is a specific polarization for the absorption which is $\hat{\boldsymbol{\epsilon}}^{\prime}$, the polarization of the Raman Stokes' lines. This spectrum is simply understood from the characteristics of the Autler-Townes splitting.

In the very high intensity limit $\left(\omega_{1}, \omega_{1}^{\prime} \gg s, \Gamma\right)$, there are two important absorbing lines at $\omega_{\mathrm{L}}+\omega_{12}$ and $\omega_{\mathrm{L}}+\omega_{32}$ corresponding to the absorption from the level $|2, n-1\rangle$ to the levels $|1, n\rangle$ and $|3, n\rangle$ (both the transition rate and the lower state population are important). The other lines, amplifying or absorbing are weaker by at least a factor $s^{2} / \Omega_{1}^{2}$.

It follows that the absorption spectrum consists mainly of two absorbing lines near frequencies $\omega_{\mathrm{L}} \pm \frac{1}{2} \Omega_{1}$ with a specific absorption polarization $\hat{\boldsymbol{\epsilon}}_{G^{\prime}}$. Consequently, the absorption spectrum vanishes if the polarization of the weak probe laser beam is the same as the strong laser beam, $\hat{\boldsymbol{\epsilon}}_{\mathrm{L}}$ (since $\hat{\boldsymbol{\epsilon}}_{\mathrm{L}}$ and $\hat{\boldsymbol{\epsilon}}_{G^{\prime}}$ are orthogonal, see \$4.2).

For intermediate intensities $\left(\Omega_{1} \sim s\right)$ there is no trap level and the six absorbing and amplifying lines are of the same order.

\section{Conclusion}

The discussion presented in this paper shows that at very high intensities the separation of the fluorescence spectrum in three groups of lines near $\omega_{\mathrm{L}}$ (Rayleigh), $\omega_{\mathrm{L}}-s$ (Raman Stokes'), $\omega_{\mathrm{L}}+s$ (Raman anti-Stokes') is no longer valid. The fluorescence spectrum consists of seven lines, the total splitting being mainly determined by $\left(\omega_{1}^{2}+\omega_{1}^{\prime 2}\right)^{1 / 2}$, i.e. by the Rabi nutation frequencies characterizing the coupling of the laser with the transitions $e \rightarrow g, e \rightarrow g^{\prime}$.

Atoms are accumulated in a well defined coherent superposition of $g$ and $g^{\prime}$ which is not coupled by the laser to $e$. This explains why the total scattered light decreases with incident light intensity.

The absorption spectrum contains mainly two absorbing lines around $\omega_{\mathrm{L}} \pm \frac{1}{2}\left(\omega_{1}^{2}+\omega_{1}^{\prime 2}\right)^{1 / 2}$. 


\section{Appendix. Evolution of the populations}

The notation is the same as in $\mathrm{I} . \Pi(t)$ is a $r$-component vector $\left\{\Pi_{i}(t) i=1, \ldots, r\right\}$. $\mathscr{T}$ is a $r \times r$ matrix defined by (see I equation (4.12))

$$
\mathscr{T}_{i j}=\Gamma_{i} \delta_{i j}-\Gamma_{i j}
$$

where $\Gamma_{i j}$ is the transition rate from the level $|j, n+1\rangle$ to the level $|i, n\rangle, \Gamma_{i}$ the total transition rate from the level $|i, n\rangle$ :

$$
\Gamma_{i}=\sum_{l} \Gamma_{l i}
$$

The evolution of the populations is described by the equation (see I equation (4.11))

$$
\left(\frac{\mathrm{d}}{\mathrm{d} t}+\mathscr{T}\right) \Pi(t)=0
$$

It appears from (A.1) and (A.2) that the sum of the $r$ lines of $\mathscr{T}$ is zero

$$
\sum_{i} \mathscr{T}_{i j}=0
$$

It follows that $\mathscr{T}$ has an eigenvalue which is 0 and which corresponds to a steady-state regime. The $(r-1)$ non-zero eigenvalues of $\mathscr{T}$ correspond to a transient regime which lasts a time determined by the weakest of them. $\Pi(t)$ can be written

$$
\Pi(t)=\Pi(+\infty)+\Pi^{\mathrm{tr}}(t)
$$

where $\Pi(+\infty)$ and $\Pi^{\operatorname{tr}}(t)$ are the steady-state and transient regime contributions to the populations.

We calculate now the quantity $\int_{0}^{T} \Pi(t) d t$ which is necessary for the evaluation of the intensities of the components of the fluorescence and absorption spectra through the expressions (5.1), (5.4) and (6.3) of I. As $T$ is supposed to be much longer than the time constants characterizing the transient regime, one can write

$$
\int_{0}^{T} \Pi(t) \mathrm{d} t=T \Pi(+\infty)+\int_{0}^{\infty} \Pi^{\mathrm{tr}}(t) \mathrm{d} t .
$$

Let us call $\tilde{\Pi}(p), \tilde{\Pi}^{\mathrm{tr}}(p)$ the Laplace transforms of $\Pi(t)$ and $\Pi^{\mathrm{tr}}(t)$. The expressions (A.3), (A.5) and (A.6) become respectively

$$
\begin{aligned}
& (p+\mathscr{T}) \tilde{\Pi}(p)=\Pi(0) \\
& \tilde{\Pi}(p)=\frac{\Pi(+\infty)}{p}+\tilde{\Pi}^{\mathrm{tr}}(p) \\
& \int_{0}^{T} \Pi(t) \mathrm{d} t=T \Pi(+\infty)+\tilde{\Pi}^{\operatorname{tr}}(0) .
\end{aligned}
$$


From (A.7) and (A.8), one deduces

$$
p \tilde{\Pi}(p)=\frac{p}{p+\mathscr{T}} \Pi(0)=\Pi(+\infty)+p \tilde{\Pi}^{\mathrm{tr}}(p)
$$

which can be written

$$
R(p) \Pi(0)=\Pi(+\infty)+p \tilde{\Pi}^{\operatorname{tr}}(p)
$$

where

$$
R(p)=\frac{p}{p+\mathscr{T}}
$$

$\tilde{\Pi}^{\operatorname{tr}}(p)$ describes the transient regime and is therefore an analytic function around $p=0$. It follows that

$$
\begin{aligned}
& \Pi(+\infty)=R(0) \Pi(0) \\
& \tilde{\Pi}^{\operatorname{tr}}(0)=\frac{\mathrm{d} R}{\mathrm{~d} p}(0) \Pi(0) \\
& \int_{0}^{T} \Pi(t) \mathrm{d} t=\left(T R(0)+\frac{\mathrm{d} R}{\mathrm{~d} p}(0)\right) \Pi(0) .
\end{aligned}
$$

Equation (A.15) allows a calculation of steady-state and transient regime contributions as functions of the initial state $\Pi(0) \dagger$.

Application to a three-level atom

In the three-level atom defined in $\$ 2$, the detailed-balance condition is fulfilled

$$
\Gamma_{i j} \Pi_{j}(+\infty)=\Gamma_{j i} \Pi_{i}(+\infty)
$$

Expressions (A.13) and (A.16) are indeed found equivalent for a three-level atom if and only if

$$
\Gamma_{12} \Gamma_{23} \Gamma_{31}=\Gamma_{13} \Gamma_{32} \Gamma_{21}
$$

and this condition (A.17) is fulfilled since the transition rates can be expressed as

$$
\Gamma_{i j}=\left|\left\langle i, n-1\left|D^{-}\right| e, n\right\rangle\right|^{2}|\langle e, n \mid j, n\rangle|^{2}
$$

which shows that the $i$ and $j$ dependences of $\Gamma_{i j}$ factorize.

The steady-state value can be evaluated more conveniently from the detailedbalance condition (A.16) than from the expression (A.13).

The transient regime contribution can be computed from the expression (A.15). † One can check that $R(0)$ is such that the steady-state value $\Pi(+\infty)=R(0) \Pi(0)$ does not depend on the initial state $\Pi(0)$. 
One gets

$$
\int_{0}^{T} \Pi_{i}^{\mathrm{tr}}(t) \mathrm{d} t=\frac{1}{\gamma_{a} \gamma_{b}}\left(\left(\gamma_{a}+\gamma_{b}\right)\left(\Pi_{i}(0)-\Pi_{i}(+\infty)\right)+\sum_{j \neq i}\left(\Gamma_{i j} \Pi_{j}(0)-\Gamma_{j i} \Pi_{i}(0)\right)\right)
$$

where the non-zero eigenvalues of $\mathscr{T}, \gamma_{a}$ and $\gamma_{b}$, only appear through their sum and product:

$$
\begin{aligned}
& \gamma_{a}+\gamma_{b}=\sum_{i} \mathscr{T}_{i i}=\sum_{i}\left(\Gamma_{i}-\Gamma_{i i}\right) \\
& \gamma_{a} \gamma_{b}=\sum_{i<j}\left(\mathscr{T}_{i i} \mathscr{T}_{j j}-\mathscr{T}_{i j} \mathscr{T}_{j i}\right) .
\end{aligned}
$$

\section{References}

Autler S H and Townes C H 1955 Phys. Rev. 100 703-22

Baklanov E V 1973 Zh. Eksp. Teor. Fiz. 65 2203-13 (Sov. Phys.-JETP 38 1100-4)

Cohen-Tannoudji C and Reynaud S 1977 J. Phys. B: Atom. Molec. Phys. 10 345-63

Mollow B R 1972 Phys. Rev. A 5 1522-7

-1973 Phys. Rev. A 8 1949-61

Reynaud S 1977 Thèse de troisième cycle Paris

Schuda F, Stroud C R Jr and Hercher M 1974 J. Phys. B: Atom. Molec. Phys. 7 L198-202

Walther H 1975 Proc. 2nd Laser Spectroscopy Conf., 1975 ed S Haroche, J C Pebay-Peyroula, T W Hansch and S H Harris (Berlin: Springer) pp 358-69

Wu F Y, Grove R E and Ezekiel S 1975 Phys. Rev. Lett. 35 1426-9 